\title{
Myocardial protection in the failing heart: II. Effect of pulsatile cardioplegic perfusion under simulated left ventricular restoration
}

\author{
Ghassan S. Kassab, PhD, ${ }^{a}$ Monica Kostelec, MD, ${ }^{b}$ Gerald D. Buckberg, MD, ${ }^{c, d}$ James Covell, MD, ${ }^{b}$
} Ahmad Sadeghi, MD, ${ }^{c, d}$ and Julien I. E. Hoffman, $M^{e}$

See related article on page 875 .
From the Department of Biomedical Engineering, University of California, Irvine ${ }^{\text {a }}$; Department of Medicine, University of California, San Diego ${ }^{\text {b }}$, Department of Surgery, David Geffen School of Medicine at University of California, Los Angeles ${ }^{c}$; Option on Bioengineering, California Institute of Technology, Pasadena ${ }^{\mathrm{d}}$; and Department of Pediatrics and Cardiovascular Research Institute, University of California, San Francisco. $^{\mathrm{e}}$

This research was supported in part by the Edward Life Sciences and by National Institutes of Health, National Heart, Lung, and Blood Institute Grant 2 R01 HL055554-06. Gerald Buckberg reports consulting fees from Sorin Biomedical, Irvine, California.

Received for publication Sept 29, 2005; accepted for publication April 11, 2006.

Address for reprints: Gerald D. Buckberg, MD, David Geffen School of Medicine at UCLA, 10833 Le Conte Avenue, 62-258 CHS, Los Angeles, CA 90095 (E-mail: gbuckberg@mednet.ucla.edu).

J Thorac Cardiovasc Surg 2006;132:884-90 $0022-5223 / \$ 32.00$

Copyright $\odot 2006$ by The American Association for Thoracic Surgery

doi:10.1016/j.jtcvs.2006.04.048
Objective: The open ventricle was studied in pacing-induced experimental heart failure to determine the extent of coronary perfusion and distribution during either continuous or pulsatile cardioplegic perfusion compared with whole blood in the beating heart.

Methods: In 5 animals that underwent pacing-induced heart failure and in 6 control swine, regional coronary blood flows were measured on bypass in the open left ventricle (simulating exposure for left ventricle restoration) during (1) beating, (2) nonpulsatile cardioplegia, and (3) pulsatile cardioplegia modalities. Mean perfusion pressure was maintained at $80 \mathrm{~mm} \mathrm{Hg}$.

Results: Flow magnitude and distribution differed in control and failing hearts in the open left ventricle. In control hearts, transmural and endocardial cardioplegic flow of nonpulsatile and pulsatile flow (which were similar to each other) exceeded beating flow by $63 \%$ and $70 \%$, respectively, in the open left ventricle condition. Transmural and subendocardial vascular resistance increased in failing hearts during cardioplegic delivery, resulting in lower subendocardial flow under nonpulsatile conditions for the same perfusion pressure. In failing hearts, subendocardial perfusion conditions did not change in the beating state $(0.89 \mathrm{vs} 0.78 \mathrm{~mL} / \mathrm{min} / \mathrm{g}$ in control and failing open beating states, respectively), but nonpulsatile cardioplegic flow was significantly reduced by $154 \%$, and became lower than beating flow by $32.2 \%(0.78$ vs $0.59 \mathrm{~mL} / \mathrm{min} / \mathrm{g}$ ). Conversely, pulsatile cardioplegic delivery improved endocardial flow in the open failing hearts, as cardioplegic perfusion with pulsatility exceeded beating flow by $41 \%$. In heart failure, pulsatility from either the beating heart, which causes extrinsic compression of coronary vessels, or intrinsic vessel distension during pulsatile cardioplegic perfusion preserved endocardial perfusion better than nonpulsatile cardioplegia at the same perfusion pressure.

Conclusion: In the failing open ventricle (simulated geometry during ventricular restoration), subendocardial blood flow was maintained in the beating state, but decreased significantly from control values during nonpulsatile cardioplegic perfusion. Conversely, pulsatile cardioplegic delivery improved subendocardial perfusion of the open failing ventricle. These findings of improved subendocardial perfusion during pulsatile delivery (either during beating or cardioplegic perfusion) compared with nonpulsatile cardioplegic delivery may have important implications for myocardial protection in failing hearts.

A basic principle of cardioplegic myocardial protection is to provide adequate distribution of the solution to all regions of the heart. This principle is especially important when reperfusion blood cardioplegia is supplied to energy-depleted, failing myocardium during open surgery. Some cardioplegic solutions lead to heterogeneity of subendocardial flow, ${ }^{1,2}$ and this may lead to patchy 


\section{Abbreviations and Acronyms}

SPSS $=$ Statistical Package for the Social Sciences

necrosis. The heterogeneous spatial distribution of blood flow $^{1,3-5}$ amounts to a 3- or 4-fold variation in blood flow from region to neighboring region. This is called the "twinkling" phenomenon. It is physiologic in the beating heart ${ }^{4,6}$ and persists after cardioplegic perfusion. ${ }^{3}$

Delivery of cardioplegia is the only instance during a cardiac operation, besides elective ventricular fibrillation, when myocardial nutritive flow is provided in nonpulsatile fashion. Unfortunately, ventricular fibrillation may impair subendocardial perfusion if there is associated coronary stenosis, ${ }^{7}$ ventricular hypertrophy,${ }^{8}$ or inotropic stimulation with catecholamines. ${ }^{9}$ Although the benefits of pulsatile flow are debated, ${ }^{10-12}$ they seem to enhance regional and transmural myocardial flow ${ }^{13}$ and metabolism ${ }^{14}$ in fibrillating hearts with critical coronary stenoses ${ }^{15,16}$ or ventricular hypertrophy. ${ }^{17,18}$ The effects of pulsatility have not been studied for cardioplegic distribution, especially in failing hearts that require left ventricular restoration treatment. Such information in heart failure procedures may help enhance cardioprotective strategies.

In a model of dilated heart failure caused by pacinginduced cardiomyopathy, ${ }^{19}$ the objective of this study was to (1) simulate operative conditions during ventricular restoration by using ventriculotomy to create the open ventricle, whereby traction creates the geometric configuration that normally exists in the working heart, and (2) evaluate the differences between coronary flow perfusion in the beating state and cardioplegia states (conventional nonpulsatile and pulsatile cardioplegia).

Our hypothesis is that phasic flow delivered either during the beating state or by infusion of pulsatile blood cardioplegia in the arrested state will increase tissue perfusion in the failing myocardium, compared with steady cardioplegic flow. Flow distribution in heart failure was compared with control conditions in the normal nonfailing heart. Our results demonstrate improved subendocardial perfusion of failing hearts under traction with phasic flow either in the beating state or under pulsatile cardioplegic conditions. The limitations and clinical implications of these results are discussed.

\section{Methods}

The experimental protocol is identical to that of the companion article. The major focus of this study was the comparison of pulsatile and nonpulsatile perfusion in the open condition (beating heart, pulsatile cardioplegia, and nonpulsatile cardioplegia). The effect of traction, between the decompressed vented heart and the open ventricle under traction, is the subject of the companion study. All hearts were studied under traction in this study. The stability of the model was verified, because mean steady-state "beating closed-state" flow returned to control levels after each microsphere study.

\section{Myocardial Blood Flow During Cardiopulmonary Bypass}

Myocardial blood flow at a perfusion pressure of $80 \mathrm{~mm} \mathrm{Hg}$ was determined with fluorescent microspheres during a randomly ordered sequence of cardiac perfusion techniques during cardiopulmonary bypass with the aorta crossclamped and the heart perfused with a Sorin perfusion system (Sorin Biomedical, Irvine, Calif). To assess the stability of each preparation, the "closed heart beating" condition was repeated at the beginning and end of each study. There were no time-related significant differences in the total coronary blood flow measured at a perfusion pressure of 80 $\mathrm{mm} \mathrm{Hg}$ with these varied perfusion sequences during bypass runs that lasted between 2.5 and 4 hours.

\section{Data and Statistical Analysis}

The flow resistance was computed as the quotient of the pressure difference along the coronary circulation and the flow. Arterial pressure was set at $80 \mathrm{~mm} \mathrm{Hg}$, and the vented heart was set at 0 venous pressure to compute the flow resistance at $80 \mathrm{~mm}$ $\mathrm{Hg} /$ flow/100 g of tissue. Data were analyzed using the Statistical Package for the Social Sciences program (SPSS Inc, Chicago, Ill) and expressed as the mean \pm standard error of the mean. A 2-way analysis of variance for repeated measures was used to determine significance.

\section{Results}

Figure 1, $A$ shows that transmural myocardial blood flow differed in control and failing hearts in relation to perfusion condition. In normal hearts, cardioplegic flow exceeded beating flow by $64 \%$ and was similar in the nonpulsatile and pulsatile states $(1.32 \mathrm{~mL} / \mathrm{min} / \mathrm{g})$. Heart failure did not significantly change flow in the beating state from control conditions, but had a principal effect on cardioplegic flow. Consequently, nonpulsatile cardioplegic flow decreased by $106 \%$ less than control levels and reached values that were slightly less than beating flow (1.32 vs $0.64 \mathrm{~mL} / \mathrm{min} / \mathrm{g})$. Pulsatile cardioplegia flow also decreased $(P<.02)$, but pulsatility provided $56 \%$ more flow than nonpulsatile cardioplegic perfusion, and thus maintained a $30 \%$ greater perfusion than the beating state. Figure $1, B$ shows the average transmural flow resistance in control and failure groups, which inversely reflects these flow changes.

The changes in average subendocardial blood flow and resistance are shown in Figure 2, $A$ and $B$ and are most pronounced in failing hearts. Beating flow remained at comparable levels during control and failure states, but nonpulsatile cardioplegic flow decreased by $154 \%$ in failing hearts $(P<.04)$, and decreased below beating flow by $32 \%$ ( 0.78 vs $0.59 \mathrm{~mL} / \mathrm{min} / \mathrm{g})$. In contrast, pulsatile cardioplegia augmented endocardial flow by $86 \%$ more than nonpulsatile cardioplegic flow. Endocardial resistance inversely reflected these flow alterations, in- 

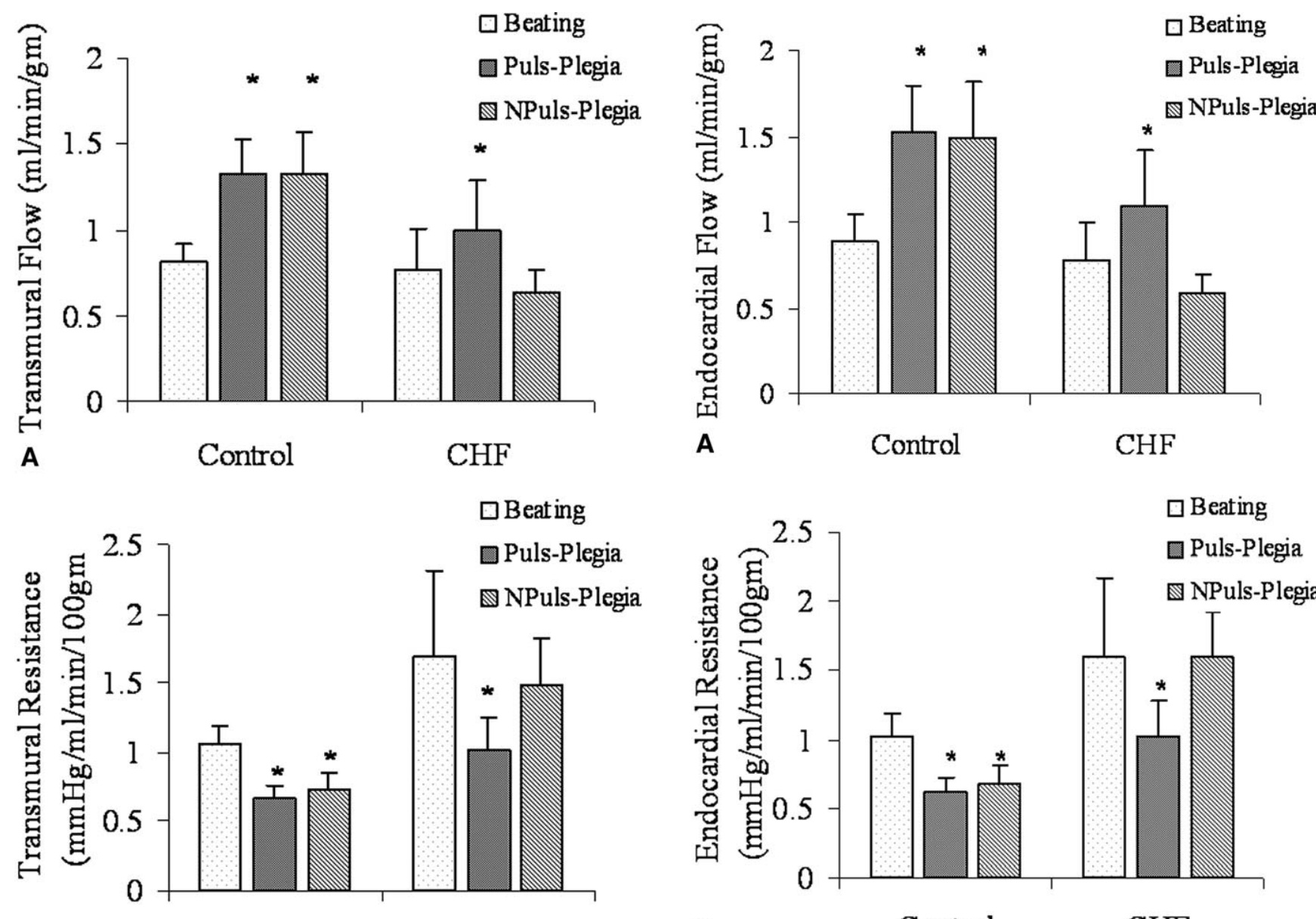

\section{B Control $\mathrm{CHF}$}

A

Control

$\mathrm{CHF}$

Figure 1. A, Mean transmural flow for control and CHF in beating open heart (traction) with whole blood perfusion (Beating), open heart pulsatile perfusion cardioplegia (PulsPlegia), and open heart nonpulsatile perfusion cardioplegia (NPuls-Plegia). B, Mean transmural resistance for the 3 groups of control and heart failure. *Statistical significance between the respective groups. CHF, Congestive heart failure.

creasing by $137 \%$ during nonpulsatile cardioplegic perfusion after failure. Pulsatile cardioplegia reduced this higher resistance in failing hearts because endocardial perfusion was better maintained.

The ratio of endo-to-epicardial blood flow distribution was approximately 1.2 in normal and failing hearts in the beating state. Preferential subendocardial perfusion occurred in control conditions as the endo-to-epicardial ratio became 1.5 (Figure 3$)$ with pulsatile $(P=.048)$ or nonpulsatile $(P=.09)$ cardioplegia. Conversely, failure decreased this ratio to 1.0 with nonpulsatile cardioplegia as endocardial perfusion was markedly curtailed $(P=.018)$, and to a lesser extent (1.2) when pulsatile cardioplegia was administered $(P=.079)$.

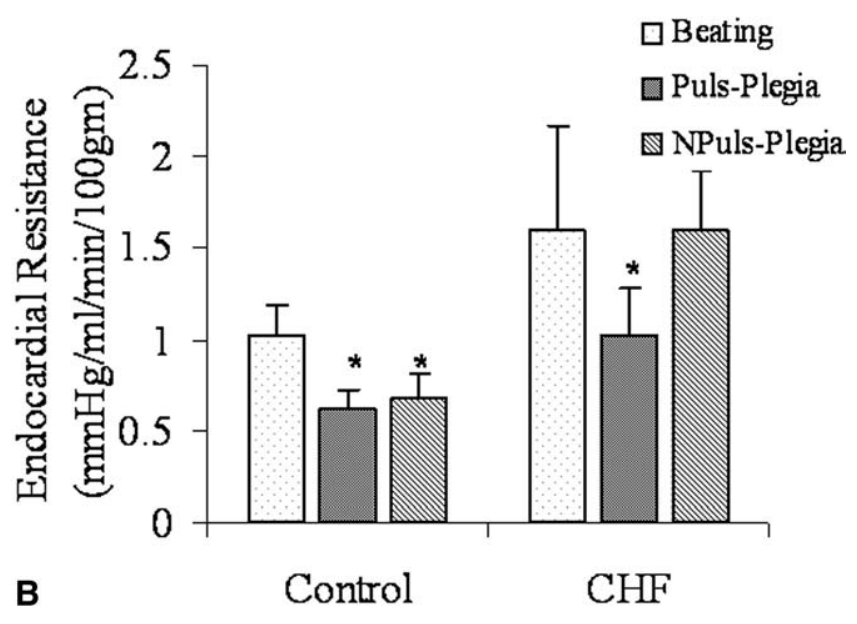

Figure 2. A, Endocardial flow for control and CHF in beating open heart (traction) with whole blood perfusion (Beating), open heart pulsatile perfusion cardioplegia (Puls-Plegia), and open heart nonpulsatile perfusion cardioplegia (NPuls-Plegia). B, Mean endocardial resistance for the $\mathbf{3}$ groups of control and heart failure. *Statistical significance between the respective groups. CHF, Congestive heart failure.

\section{Discussion}

This study evaluated transmural myocardial flow in open ventricles in normal control conditions and in failing hearts after pacing-induced cardiomyopathy to simulate the operative conditions during ventricular restoration for heart failure. The perfusion effects of the beating heart were compared with nonpulsatile and pulsatile blood cardioplegia as the preferred method of protection. ${ }^{23,24}$ The present chronic heart failure model simulated the geometric conditions of an open ventricle whose edges are kept under traction during ventricular restoration for dilated cardiomyopathy. The chronic model of heart failure is essential as negligible changes occurred in control hearts. Coronary vascular resistance increased during heart failure, resulting in impaired 


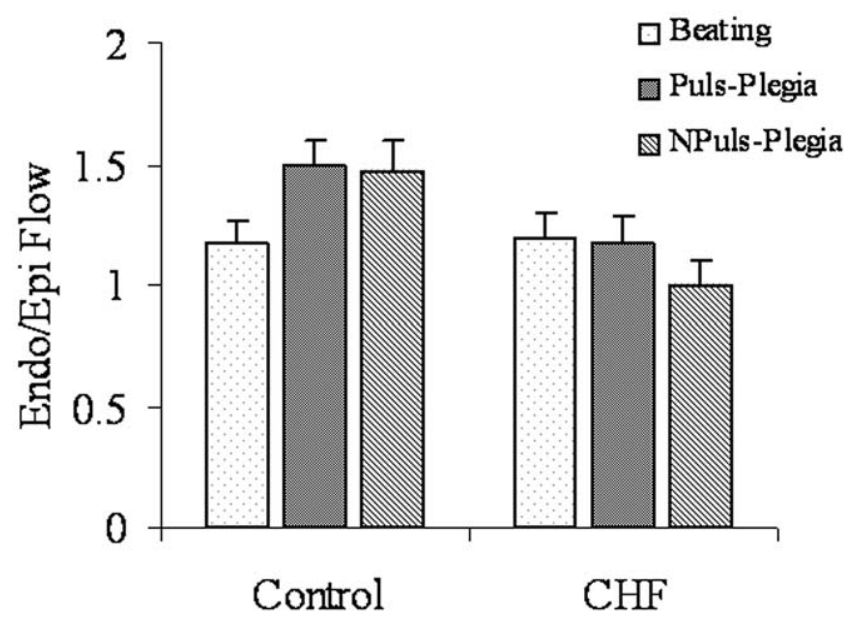

Figure 3. Epicardial to endocardial (Epi/Endo) blood flow ratio for control and CHF in open heart beating with whole blood perfusion, open heart pulsatile perfusion cardioplegia, and open heart (traction) nonpulsatile perfusion cardioplegia. *Statistical significance between the respective groups. CHF, Congestive heart failure.

endocardial perfusion during delivery of warm nonpulsatile cardioplegia delivery. We suspect that vascular resistance would become further enhanced during cold cardioplegia delivery.

Our hypothesis was that pulsatile perfusion enhances flow in failing ventricles, either in the beating failing heart or in the pulsatile infusion of the warm blood cardioplegia solution compared with the conventional delivery of continuous flow cardioplegia. Although no ischemia was imposed, lactate production measurements were not made to confirm metabolic evidence of the absence of underperfusion. However, metabolic baseline flow in the "beating closed state" always returned to pre-ventriculotomy levels following before and after randomization of delivery methods at $80 \mathrm{~mm} \mathrm{Hg}$ perfusion pressure in all studies, which implies that ischemia did not occur.

Minimization of intraoperative damage is a principal objective during every cardiac operation, and this goal is especially important is failing hearts, which are more vulnerable to injury. To that end, numerous methods of intraoperative myocardial management have been developed, ${ }^{22}$ and blood cardioplegia has become the preferred cardioprotective strategy. ${ }^{23,24}$ In the present study, we used continuous warm whole blood perfusion (without cardioplegia) or warm blood cardioplegia solution administered in either a pulsatile or nonpulsatile fashion to determine the proper perfusion technique in the failing heart. Superimposition of ischemia (which was avoided) would accentuate underperfusion during intermittent doses.

\section{Experimental Model}

A fundamental component of this study was the evaluation of perfusion methods in a dilated failing ventricle, which was studied in the open ventricular state to mirror events during ventricular restoration. Pacing causes a markedly dilated cardiomyopathy ${ }^{20}$ with right and left ventricular wall thinning without hypertrophy or increase in heart weight. ${ }^{20,21}$ This model provides functional and neurohormonal changes ${ }^{25}$ that are relevant in the study of heart failure. Furthermore, the increased ventricular volume and spherical shape closely correspond to the size and shape of hearts undergoing ventricular restoration. Helmer and colleagues ${ }^{21}$ studied regional differences in endocardial flow and suggest that alterations in coronary vascular structure and function may accompany left ventricular remodeling during the progression of heart failure. The study model of empty hearts on bypass in the open state may be important, because Neumann and $\mathrm{Heusch}^{26}$ studied working dilated hearts and did not find preferential impairment of subendocardial blood flow either at rest or during exercise in dogs paced to failure. In contrast, our studies of open dilated hearts on bypass show that flow is redistributed away from the subendocardium in the failure state.

Failing hearts without bypass are vulnerable to inadequate protection, because pacing-induced congestive heart failure is associated with diminished myocardial blood flow reserve $^{27}$ that may be selective in the endocardium ${ }^{28}$ as a result of coronary endothelial dysfunction. ${ }^{29}$ Reduced myocardial blood flow at rest has been reported in conscious dogs and in pigs with pacing-induced heart failure. ${ }^{27,28}$ The pacing stimulus was stopped preoperatively in these studies, yet the open failing hearts on bypass decreased mean blood flow and especially subendocardium perfusion only when nonpulsatile cardioplegia was used for protection. Conversely, delivery of either pulsatile flow in the beating heart without cardioplegia or the use of pulsatile cardioplegia avoided subendocardial underperfusion.

Krombach and colleagues ${ }^{25}$ established the flow liability of failing dilated hearts without bypass, and suggested that increased coronary vascular resistance was important because there was no physical blood vessel obstruction. Our study of normal and failing hearts in the open state on bypass exhibited steady-state reduction of blood flow only during cardioplegic perfusion, because beating flow was similar in the control and failure states (Figure 1). This observation underscores the importance of using models that closely reflect clinical conditions, especially because failure causes selective subendocardium impairment in coronary reserve. ${ }^{28}$ Furthermore, vascular remodeling occurs in failing hearts as reflected by the lower subendocardial perfusion compared with normal hearts at the same perfusion pressure. 
For these reasons, myocardial perfusion differed between control and failure cohorts. In control studies during the beating state, transmural and endocardial flow were less than after nonpulsatile or pulsatile cardioplegic infusion. Beating perfusion was similar in the control and failing hearts when studied under traction. Conversely, nonpulsatile subendocardial cardioplegia flow decreased by $137 \%$ in failing hearts during ventricular traction (Figure 1). In contrast, perfusion increased by $86 \%$ when pulsatile cardioplegia was used in failing hearts, and thereby resulted in higher flows than were observed during beating flow measurements that were recorded with or without pacing-induced heart failure. The model of heart failure and the role of providing a pulsatile form of delivery are factors that must be considered during the development of protection methods in failing dilated hearts.

\section{Possible Mechanisms}

In heart failure, the differences in flow between beating and cardioplegia are somewhat diminished (Figures 1, $A$ and $2, A)$ compared with control hearts. This finding may reflect remodeling of geometric and resistance elements on perfusion characteristics because vascular resistance increased in failing hearts compared with control hearts (Figures $1, B$ and $2, B$ ) perfused at the same coronary pressure. The increase in resistance may stem from reduction in lumen diameters, increased length, or decreased numbers of resistance vessels. Each hypothesis is reasonable because chamber size increases and stress in the myocardium (external to the blood vessels) increases significantly in dilated failure as predicted by Laplace's equation (larger radius and smaller wall thickness of ventricle). If the microvessel diameter is decreased, the higher peak pressure attained in the pulsatile perfusion will "open up" or recruit closed vessels, thereby increasing flow as resistance decreases.

The endocardial effect may be more pronounced during nonpulsatile cardioplegic perfusion, leading to the observed reduction (compared with control) in preferential endocardial perfusion resulting in a more uniform endo-to-epicardial ratio (near unity) in heart failure (Figure 3). The perfusion ratios can be misleading, because the normal heart has preferential subendocardial perfusion (endo-to-epicardial ratio of 1.4) during cardioplegic delivery, and the ratio decreased to unity $(\sim 1.0)$ in the failing open heart during nonpulsatile cardioplegic perfusion. Although metabolic measurements were not made, we previously observed ischemia during similar decreased in flow ratios in hypertrophied hearts subjected to ventricular fibrillation or in normal hearts given an electrical fibrillation stimulus. ${ }^{2,3}$ In contrast, this study was performed without ischemia, which usually is a normal clinical component of cardioplegic delivery during intermittent delivery. Consequently, periodic pulsatile cardioplegia would improve subendocardial perfusion compared with nonpulsatile cardioplegia.

Studies of flow variability in the autoregulating vascular bed are difficult to interpret, because both spatial and temporal variability exist. For example, abolishing autoregulation during reactive hyperemia opens new beds; the 6-fold increase in flow during this response defines a new perfusion snapshot by spatially adding new vascular beds in the same time interval. Conversely, maximal vasodilation creates non-autoregulating beds that more clearly display spatial or vascular inadequacies. The variability in the spatial distribution of cardioplegia was reported by Aldea and colleagues ${ }^{1}$ in maximally dilated normal hearts and did not address the issue of flow pulsatility in the arrested state. Our report evaluates sanguineous cardioplegic flow to failing myocardium with intact, autoregulating microvasculature under traction. Such traction is essential to expose the myocardium for repair and simultaneously restores the normal curvature of the heart compared with the shrunken state existing during venting in the closed state.

\section{Role of Pulse Pressure}

Goto and colleagues ${ }^{30}$ and Sorop and colleagues ${ }^{31}$ reported that increased pulse pressure causes vasodilation in ex vivo experiments on small epicardial and endocardial coronary vessel preparations independent of flow (no flow conditions). The vasodilatory effect of pulse pressure was endothelium-independent because denudation did not abolish the response. This suggests that flow pulsation may simulate luminal changes of resistance vessels normally observed during muscular compression resulting from cardiac contraction. We suspect that varying pulsation either by intermittent muscular closure during beating or by introduction of pulsatile vessel opening during cardioplegia may favor vasodilatation to increase perfusion, but this hypothesis remains to be further tested in future studies.

The majority of the mechanisms of enhanced perfusion with pulsation indicate a central role of precapillary sphincters and capillaries ${ }^{6,10,11}$ in translating the energy of the pulse into a more efficient flow, that is, to keep more vessels open. These vascular elements probably supply areas of muscle smaller than the 1-g samples used in the present study. Consequently, a more precise method of myocardial sampling may be needed to accurately elucidate the microcirculatory changes.

In the beating heart, the transmural pressure is larger in the endocardial vessels, which may lead to their preferential dilation. Although the mechanism of pulse pressure-induced vessel dilatation remains unknown, there may be significant vascular remodeling in heart failure. Furthermore, pulsatility of the cardioplegic solution may be small compared with the phasic nature of blood flow in the control beating heart, because the cardioplegic perfused vessel remains full, in 
contrast with the periodic emptying that follows each myocardial contraction.

Streeter and colleagues ${ }^{32}$ and LeGrice and colleagues ${ }^{33}$ reported an increase in obliqueness of fibers (cleavage planes) in dilated hearts, whereas the force of contraction was reduced. The same vessels remain open during cardioplegia, and there may be increased luminal patency because the extravascular forces that empty vessels during contraction are absent. More important, these changes may more than offset the "wastage" of nutritive perfusion that exists in the beating heart when there is intermittent opening and closing of vessels that limit nutritive perfusion, because conductance vessel refilling must occur as the heart beats in a decompressed vented state. In heart failure there is a greater tendency for vessel closure, as reflected by the increased resistance. Although the same vessels remain open during cardioplegia, there is absence of contractility, so there may be recruitment of some of the closed vessels during pulsatile perfusion. We cannot distinguish this from vasodilation, because we only have the flow recordings in this observational study and did not offset the autoregulatory response by maximal vasodilation. ${ }^{2}$

Our study documents two main observations in its examination of the effect of perfusion techniques in failing hearts: (1) Conventional nonpulsatile cardioplegia diminishes flow, and (2) delivering pulsatile flow is important, whether in the beating empty state or by augmenting cardioplegia in a pulsatile fashion. Steed and colleagues ${ }^{18}$ showed that pulsatile augmentation in this state improved endocardial distribution during ventricular fibrillation. Bixler and colleagues ${ }^{17}$ showed that perfusion at moderate pressures improved flow to all layers of hypertrophied myocardium. In addition, hypertrophied hearts have smaller ventricular volumes, and thus are more likely to have subendocardial vascular distortion. ${ }^{9}$ Those hearts may be particularly suited for pulsatile delivery of protective cardioplegia because this technique may be more effective at distending the compressed microvasculature, which provides increased cardioplegic flow that is particularly critical for the subendocardium. This same concept may be directly applicable to the delivery of pulsatile cardioplegia to infant hearts with comparatively small ventricular volumes. Subendocardial vascular distortion may be partially accountable for the mitochondrial damage and edema formation recently reported after delivery of cardioplegia ${ }^{34}$ in a neonatal population.

These aforementioned observations suggest that (1) pulsation may be a counterforce to muscular compression of coronary vessels in a beating or fibrillating heart; (2) systolic myocardial compression may close and open vessels intermittently and favor "twinkling" differences as altering regions become perfused in the nonmaximally dilated state; (3) no intermittent opening/closing of vessels can occur with cardioplegic arrest, so that pulsation may force open some closed regions to improve the spatial effects of twinkling and avoid an otherwise constant perfusion to only open vessels; and (4) geometric changes may occur in the vented ventricle that causes vessel distortion compared with the normal architecture of the ejecting ventricle. This may be exaggerated when the vessel wall/lumen ratio changes in heart failure with decompression during a vent; pulsatility may force open these closed vessels and improve perfusion. This benefit may be better than only increasing mean perfusion pressure without a pulsatile effect because pulsatility improves total and regional endocardial flow. Testing of these various hypotheses is the task of future studies. Although the benefits of pulsatile cardioplegia are evident, further validation may highlight an innovative method that improves myocardial protection in high-risk patients. ${ }^{19,35}$

We acknowledge the contributions of staff at all 3 institutions and in particular the surgical and administrative skills of $\mathrm{Mr}$. Richard Pavelec, without whose contributions these complex studies would not have been possible.

\section{References}

1. Aldea GS, Austin RE Jr, Flynn AE, Coggins DL, Husseini W, Hoffman JIE. Heterogeneous delivery of cardioplegic solution in the absence of coronary artery disease. J Thorac Cardiovasc Surg. 1990;99:345-53.

2. Ferrans VJ. Morphological methods for evaluation of myocardial protection. Ann Thorac Surg. 1975;20:11-20.

3. Marcus ML, Kerber RE, Ehrhardt J, Abboud FM. Three dimensional geometry of acutely ischemic myocardium. Circulation. 1975; 52:254-63.

4. Marcus ML, Kerber RE, Erhardt JC, Falsetti HL, Davis DM, Abboud FM. Spatial and temporal heterogeneity of left ventricular perfusion in awake dogs. Am Heart J. 1977;94:748-54.

5. Falsetti HL, Carroll RJ, Marcus ML. Temporal heterogeneity of myocardial blood flow in anesthetized dogs. Circulation. 1975;52:848-53.

6. Bassingthwaighte JB, Malone MA, Moffett TC, King RB, Little SE, Link JM, et al. Validity of microsphere depositions for regional myocardial flows. Am J Physiol. 1987;253(1 Pt 2):H184-H93.

7. Brazier JR, Cooper N, McConnell DH, Buckberg GD. Studies of the effects of hypothermia on regional myocardial blood flow and metabolism during cardiopulmonary bypass. III. Effects of temperature, time, and perfusion pressure in fibrillating hearts. J Thorac Cardiovasc Surg. 1977;73:102-9.

8. Hottentrott CE, Towers B, Kurkji HJ, Maloney JV, Buckberg GD. The hazard of ventricular fibrillation in hypertrophied ventricles during cardiopulmonary bypass. J Thorac Cardiovasc Surg. 1973;66:742-53.

9. Utley JR, Michalsky GB, Mobin-Uddin K, Bryant LR. Subendocardial vascular distortion at small ventricular volumes. J Surg Res. 1974;17: 114-24.

10. Mavroudis C. To pulse or not to pulse. Ann Thorac Surg. 1978;25: 259-71.

11. Hickey PR, Buckley MJ, Philbin DM. Pulsatile and nonpulsatile cardiopulmonary bypass: review of a counterproductive controversy. Ann Thorac Surg. 1983;36:720-37.

12. Taylor KM. Pulsatile perfusion. In: Taylor KM, ed. Cardiopulmonary bypass principles and management. London: Chapman and Hill; 1986: 85-115.

13. Moores WY, Hannon JP, Crum J, Willford D, Rodkey WG, Geasling JW. Coronary flow distribution and dynamics during continuous and pulsatile extracorporeal circulation in the pig. Ann Thorac Surg. 1977; 24:582-90.

14. Habal SM, Weiss MB, Spotnitz HM, Parodi EN, Wolff M, Cannon PJ, et al. Effects of pulsatile and nonpulsatile coronary perfusion on 
performance of the canine left ventricle. J Thorac Cardiovasc Surg. 1976;72:742-55.

15. Schaff HV, Ciardullo RC, Flaherty JT, Brawley RK, Gott VL. Regional ischemia distal to a critical coronary stenosis during prolonged fibrillation-improvement with pulsate perfusion. Circulation. 1977; 56(3 Suppl):II25-32.

16. Ciardullo RC, Schaff HV, Flaherty JT, Donahoo JS, Gott VL. Comparison of regional myocardial blood flow and metabolism distal to a critical coronary stenosis in the fibrillating heart during alternate periods of pulsatile and nonpulsatile perfusion. $J$ Thorac Cardiovasc Surg. 1978;75:193-205.

17. Bixler TJ, Magee PG, Flaherty JT, Gardner TJ, Gott VL. Beneficial effects of pulsatile perfusion in the hypertrophied ventricle during ventricular fibrillation. Circulation. 1979;60(2 Pt 2):141-6.

18. Steed DL, Follette DM, Foglia R, Maloney JV, Buckberg GD. Effects of pulsatile assistance and nonpulsatile flow on subendocardial perfusion during cardiopulmonary bypass. Ann Thorac Surg. 1978;26:133-41.

19. Kostelec M, Covell J, Buckberg GD, Sadeghi A, Hoffman JIE, Kassab GS. Myocardial protection in the failing heart: I. Effect of cardioplegia and the beating state under simulated left ventricular restoration. J Thorac Cardiovasc Surg. 2006;132:875-83.

20. Shinbane JS, Wood MA, Jensen DN, Ellenbogen KA, Fitzpatrick AP, Scheinman MM. Tachycardia-induced cardiomyopathy: a review of animal models and clinical studies. J Am Coll Cardiol. 1997;29:709-15.

21. Helmer GA, McKirnan D, Shabetai R, Boss GR, Ross J Jr, Hammond K. Regional deficits of myocardial blood flow and function in left ventricular pacing-induced heart failure. Circulation. 1996;94:2260-7.

22. Buckberg GD. Update on current techniques of myocardial protection. Ann Thorac Surg. 1995;60:805-14.

23. Buckberg GD. Oxygenated cardioplegia: blood is a many splendored thing. Ann Thorac Surg. 1990;50:175-7.

24. Rosenkranz ER, Okamoto F, Buckberg GD, Robertson JM, VintenJohansen J, Bugyi H. Safety of prolonged aortic clamping with blood cardioplegia. III. Aspartate enrichment of glutamate-blood cardioplegia in energy depleted hearts after ischemic and reperfusion injury. J Thorac Cardiovasc Surg. 1986;91:428-35.
25. Krombach RS, Clair MJ, Hendrick JW, Houck WV, Zellner JL, Kribbs $\mathrm{SB}$, et al. Angiotensin converting enzyme inhibition, AT1 receptor inhibition, and combination therapy with pacing-induced heart failure: effects on left ventricular performance and regional blood flow patterns. Cardiovasc Res. 1998;38:631-45.

26. Neumann T, Heusch G. Myocardial, skeletal muscle, and renal blood flow during exercise in conscious dogs with heart failure. Am J Physiol. 1997;273(5 pt 2):H2452-H7.

27. Spinale FG, Tanaka R, Crawford FA, Zile MR. Changes in myocardial blood flow during development and recovery from tachycardiainduced cardiomyopathy. Circulation. 1992;85:717-29.

28. Shannon RP, Komamura K, Shen YT, Bishop SP, Vatner SF. Impaired regional subendocardial coronary flow reserve in conscious dogs with pacing-induced heart failure. Am J Physiol. 1993; 265(3 Pt 3):H801-H9.

29. Wang J, Seyedi N, Xu XB, Wolin MS, Hintze TH. Defective endothelium-mediated control of coronary circulation in conscious dogs after heart failure. Am J Physiol. 1994;266(2 Pt 2):H670-H80.

30. Goto M, VanBavel E, Giezeman MJ, Spaan JA. Vasodilatory effect of pulsatile pressure on coronary resistance vessels. Circ Res. 1996;79: 1039-45.

31. Sorop O, Spaan JA, VanBavel E. Pulsation-induced dilation of subendocardial and subepicardial arterioles: effect on vasodilator sensitivity. Am J Physiol Heart Circ Physiol. 2002;282:H311-9.

32. Streeter DD Jr, Spotnitz HM, Patel DP, Ross J Jr, Sonnenblick EH. Fiber orientation in the canine left ventricle during diastole and systole. Circ Res. 1969;24:339-47.

33. LeGrice IJ, Takayama Y, Covell JW. Transverse shear along myocardial cleavage planes provides a mechanism for normal systolic wall thickening. Circ Res. 1995;77:182-93.

34. Sawa Y, Matsuda H, Shimazaki Y, Hirose H, Kadoba K, Takami H, et al. Ultrastructural assessment of the infant myocardium receiving crystalloid cardioplegia. Circulation. 1987;76(5 Pt 2):V141-V5.

35. Hottentrott CE, Towers B, Kurkji HJ, Maloney JV Jr, Buckberg GD. The hazard of ventricular fibrillation in hypertrophied ventricles during cardiopulmonary bypass. J Thorac Cardiovasc Surg. 1973;66:742-53. 